\title{
Use of three-dimensional time-resolved phase-contrast magnetic resonance imaging with vastly undersampled isotropic projection reconstruction to assess renal blood flow in a renal cell carcinoma patient treated with sunitinib: a case report
}

\author{
Tatsuya Takayama ${ }^{*}$, Yasuo Takehara², Masataka Sugiyama², Takayuki Sugiyama', Yasuo Ishii', Kevin E Johnson ${ }^{3}$,
} Oliver Wieben ${ }^{3}$, Tetsuya Wakayama ${ }^{4}$, Harumi Sakahara ${ }^{2}$ and Seiichiro Ozono ${ }^{1}$

\begin{abstract}
Background: New imaging modalities to assess the efficacy of drugs that have molecular targets remain under development. Here, we describe for the first time the use of time-resolved three-dimensional phase-contrast magnetic resonance imaging to monitor changes in blood supply to a tumor during sunitinib treatment in a patient with localized renal cell carcinoma.
\end{abstract}

Case presentation: A 43-year-old Japanese woman with a tumor-bearing but functional single kidney presented at our hospital in July 2012. Computed tomography and magnetic resonance imaging revealed a cT1aNOM0 renal cell carcinoma embedded in the upper central region of the left kidney. She was prescribed sunitinib as neoadjuvant therapy for 8 months, and then underwent partial nephrectomy. Tumor monitoring during this time was done using time-resolved three-dimensional phase-contrast magnetic resonance imaging, a recent technique which specifically measures blood flow in the various vessels of the kidney. This imaging allowed visualization of the redistribution of renal blood flow during treatment, and showed that flow to the tumor was decreased and flows to other areas increased. Of note, this change occurred in the absence of any change in tumor size.

Conclusion: The ability of time-resolved three-dimensional phase-contrast magnetic resonance imaging to provide quantitative information on blood supply to tumors may be useful in monitoring the efficacy of sunitinib treatment.

Keywords: Renal cell carcinoma, Magnetic resonance, Phase contrast imaging, Flow analysis, Sunitinib

\section{Background}

The introduction of drugs targeting specific molecules has markedly changed the treatment of advanced renal cell carcinoma (RCC) [1-4]. Drugs currently approved in Japan include tyrosine kinase inhibitors (TKIs) such as sorafenib, sunitinib and axitinib, and mammalian target of rapamycin (mTOR) inhibitors, including everolimus

\footnotetext{
* Correspondence: ttakayam@hama-med.ac.jp

1Departments of Urology, Hamamatsu University School of Medicine, 1-20-1

Higashi-ku, Handayama, Hamamatsu, Shizuoka 431-3192, Japan

Full list of author information is available at the end of the article
}

and temsirolimus. With ongoing improvement in the resolution of imaging modalities, including multidetector row computed tomography $(\mathrm{CT})$ and high-tesla magnetic resonance imaging (MRI), more small renal masses are diagnosed incidentally, and surgical management has shifted from radical toward partial nephrectomy. Moreover, the extended use of TKIs and mTOR inhibitors to reduce primary tumor volume, followed by application of neoadjuvant therapy, is now widely used as an organsparing strategy for RCC patients. In addition, drugs that have molecular targets are increasingly assessed using 


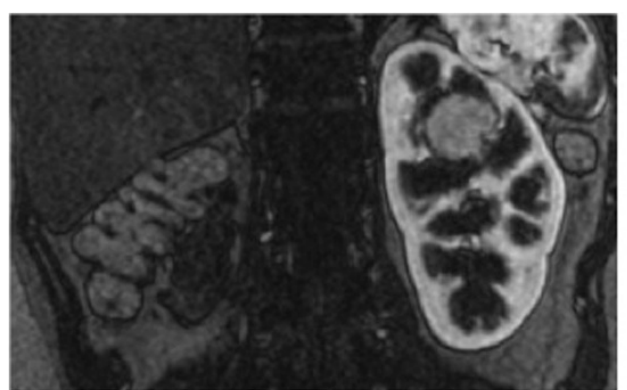

Early phase

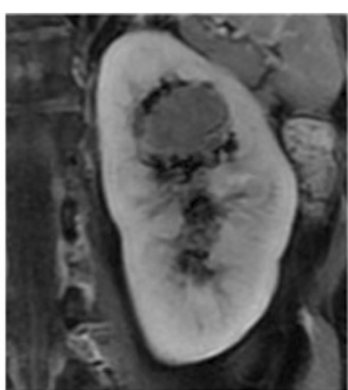

Late phase

Figure 1 Contrast-enhanced coronal T1-weighted imaging with fat saturation (left: arterial phase; right: late phase) reveals a hypervascular solitary solid mass in the upper half of the left (functional) kidney.

the Choi criteria [5], and novel criteria that can be applied after evaluation with fluorodeoxyglucose-positron emission tomography [6,7]. To date, however, few imaging modalities have been used in this context. To our knowledge, this paper is the first to show that 3D PC VIPR can be used to monitor changes in blood supply to a tumor during drug treatment.

\section{Case presentation}

A 43-year-old Japanese woman with a tumor-bearing but functional single left kidney presented at our hospital in July 2012. Her past history and family history were unremarkable in the present context. CT and MRI revealed a cT1aNOM0 RCC with maximum scores in preoperative aspects, anatomical Preoperative Aspects and Dimensions Used for an Anatomical (PADUA) criteria, and the radius exophytic/endophytic nearness anterior/posterior location (RENAL) criteria used to grade renal neoplasms (Figure 1). The tumor was embedded in the upper central region of the kidney. Because she had only a single functioning kidney, only partial nephrectomy was appropriate. However, the tumor location suggested the possibility of severe complications even after partial nephrectomy. We therefore commenced treatment with sunitinib as neoadjuvant therapy, and used MRI to monitor her condition.

The MRI modalities employed ECG-gated, respirationcompensated, time-resolved respiratory-gated threedimensional (3D) phase-contrast (PC) MRI featuring PC-VIPR (phase-contrast vastly undersampled isotropic projection reconstruction) [8]. 3D PC VIPR was performed using a 3.0 Tesla (T) MR scanner (750 Discovery, GEHC, WI) fitted with a 16-channel torso phased-array coil (GEHC), covering the entire left renal artery and parent artery (i.e. abdominal aorta) at the same level. Fast GRE-based sequence was used with the following parameters: field of view (FOV) $32 \mathrm{~cm}$, slice thickness $10 \mathrm{~mm}$, locs/slab 8, repetition time (TR) $7.0-7.1 \mathrm{~ms}$,
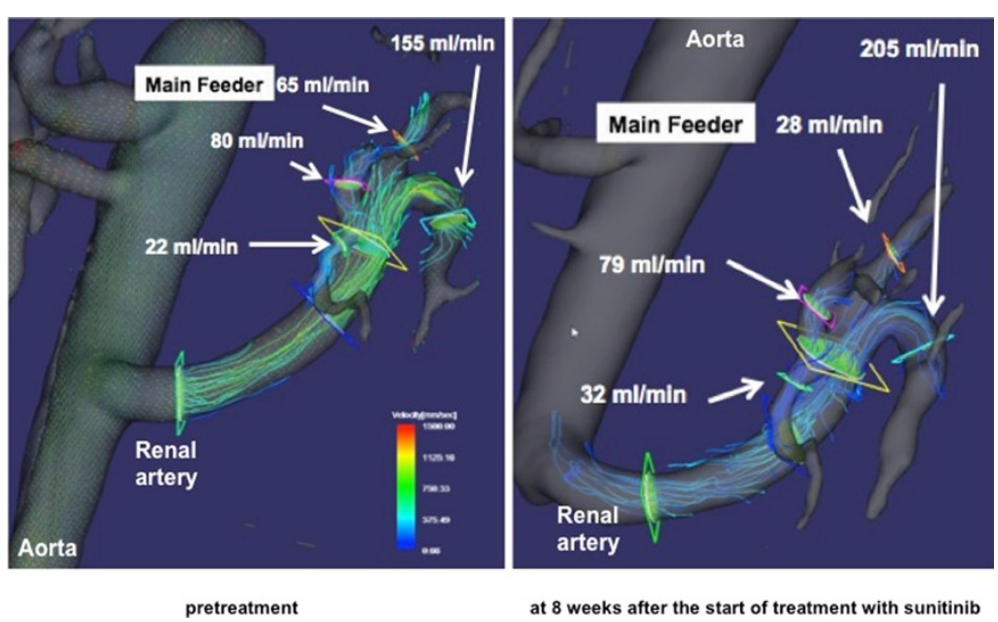

Figure 2 Blood flow in arterial branches visualized using time-resolved three-dimensional phase-contrast magnetic resonance imaging. Flows are visualized as streamlines. The regions of branches where flows were measured are shown. Blood flow in the main feeder to the tumor was $65 \mathrm{ml} / \mathrm{min}$ (left) before sunitinib treatment, but decreased to $28 \mathrm{ml} / \mathrm{min}$ after 8 weeks of sunitinib treatment (right). Of note, flow rates in the other branches increased simultaneously, which may indicate flow redistribution from the tumor to other kidney segments. 
echo time (TE) 3.3-3.4 ms, flip angle (FA; degrees) $8^{\circ}$, matrix $256 \times 256$, receiver bandwidth $62.5 \mathrm{kHz}$, partition thickness $1.25 \mathrm{~mm}$, NEX 1, number of phases/location 20, imaging time 9:29-9:36 min, respiratory window 50\%, number of projections 10,000, and encoding velocity (VENC) $100-200 \mathrm{~cm} / \mathrm{s}$ depending on the maximum velocities measured in preceding 2D cine PC studies. The 4D datasets were post-processed using flow analysis software (flova; R's Tech Co., Hamamatsu, Japan). A valuable feature of 3D PC VIPR is its capacity to delineate renal branches and obtain flow velocity data without administration of contrast medium (Figure 2).

Figure 3 illustrates the clinical course of the patient. Sunitinib was administered at the recommended dose ( $50 \mathrm{mg}$ daily; 4 weeks on followed by 2 weeks off) in the first course and at $75 \%$ relative dose intensity in the second. Tumor size and density measured on CT decreased slightly, and disease status was stable according to
Response Evaluation Criteria in Solid Tumors version 1.0 (RECIST v1.0). Tumor angiogenicity was disrupted by sunitinib, and renal blood flow was redistributed with a decrease in blood flow to the tumor and increase to other areas. Platelet concentration gradually decreased and she was classified as grade 3 under the Common Terminology Criteria Adverse Events version 3.0 system. She also developed grade 2 hypertension, grade 2 handfoot skin reactions, grade 1 dysphonia, grade 1 proteinuria, and grade 2 hypothyroidism. Sunitinib treatment was thus modified to four cycles of 2 weeks on sunitinib (37.5 mg daily) followed by a 2-week drug holiday in an effort to restore platelet levels and achieve resolution of other adverse events. However, blood flow to the tumor and tumor size reverted to and remained at levels before sunitinib treatment (Figure 3). We judged that sunitinib was unlikely to yield further useful effects, and treatment was discontinued. The patient then underwent partial

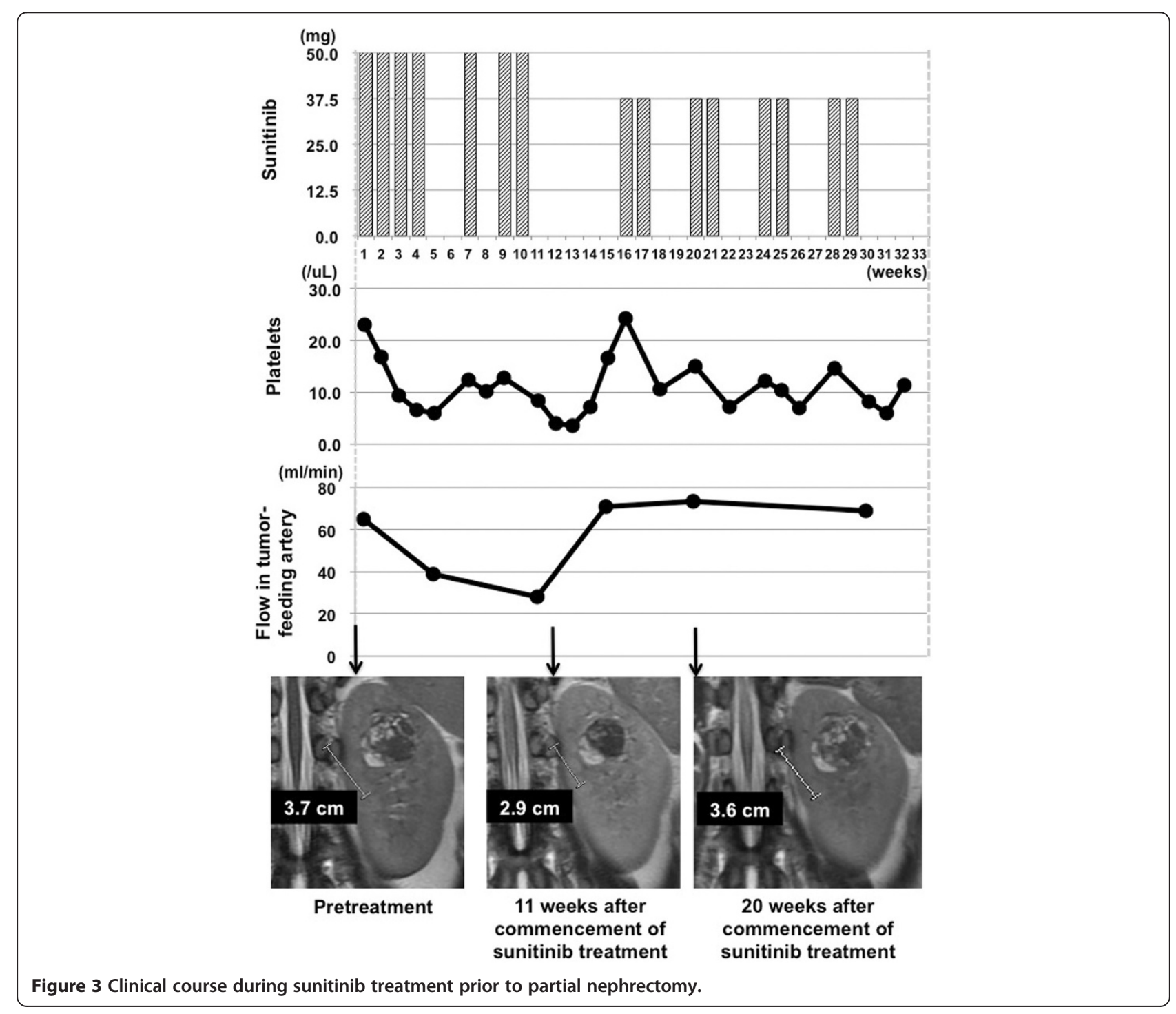


nephrectomy 1 month later. We could not find any tumor necrosis or hemorrhage reflecting the effect of sunitinib treatment pathologically. Both the operative and postoperative courses were uneventful, and she remains in good condition, with no tumor recurrence, an estimated glomerular filtration rate of $47 \mathrm{ml} / \mathrm{min} / 1.73 \mathrm{~m}^{2}$, and grade 2 hypothyroidism.

\section{Conclusion}

3D PC VIPR is a recently developed phase-contrast MR technique which uses a new k-space trajectory. A major advantage of 3D PC VIPR is its ability to both morphologically image the renal arteries and veins, and measure their blood flow velocity. Use of flow analysis software then permits flow streamlines to be visualized. A second major advantage of 3D PC VIPR is that it does not require contrast medium for renal artery depiction. Many patients with renal neoplasia have impaired renal function, and clinicians are now reluctant to administer gadolinium chelate contrast media to these patients due to the risk of nephrogenic systemic fibrosis [9].

Here, we describe a patient with RCC who underwent partial nephrectomy 8 months after the commencement of sunitinib as neoadjuvant therapy with monitoring using 3D PC VIPR. Of note, total renal arterial flow level barely changed over the entire course of treatment. Sunitinib (one course of the full recommended dose and a second course at $75 \%$ of that dose) reduced blood flow to the tumor and increased flow to other areas of the kidney. When sunitinib was recommenced after cessation to allow recovery from adverse effects, however, it did not induce the same redistribution of blood flow as it had before cessation. It is notable that the redistribution of renal blood flow might be associated with the induction of stable disease, as identified using the RECIST criteria. Although it is unclear whether this was caused by the reduction in sunitinib dose or acquisition of drug resistance by the tumor, we emphasize that 3D PC VIPR was markedly useful in monitoring therapeutic response as it allowed the measurement of blood flow in the tumor feeder vessel. These findings suggest that we should have conducted partial nephrectomy without additional administration of sunitinib at any time from 20 weeks onward.

One limitation of 3D PC-VIPR is that it is difficult to measure blood flow in very small arteries, such as tertiary vessels or smaller, using prescribed velocity encoding.

Non-contrast 3D PC VIPR revealed the redistribution of renal blood flow in a patient with RCC treated with sunitinib, including that to the tumor, without any change in tumor size. This modality may be useful in predicting the efficacy of sunitinib in terms of blood flow redistribution, and in determining surgical timing. Further evaluation in a large number of cases is warranted.

\section{Consent}

Written informed consent was obtained from the patient for publication of this case report and any accompanying images. A copy of the written consent is available for review by the Editor of this journal.

\section{Abbreviations}

RCC: Renal cell carcinoma; TKIs: Tyrosine kinase inhibitors; mTOR: Mammalian target of rapamycin; CT: Computed tomography; MRI: Magnetic resonance imaging; 3D: Three-dimensional; PC: Phase-contrast; PC-VIPR: Phase-contrast vastly undersampled isotropic projection reconstruction; RECIST v1.0:

Response Evaluation Criteria in Solid Tumors version 1.0.

\section{Competing interests}

Wakayama T is in the employ of GE Healthcare. Johnson $\mathrm{K}$ and Wieben $\mathrm{O}$ have received research funding from GE Healthcare. Takayama T, Takehara Y, Sugiyama M, Sugiyama T, Ishii Y, Sakahara H, and Ozono S have no competing interests.

\section{Authors' contributions}

$\Pi T$ and $Y T$ designed the study, acquired clinical and imaging data, analyzed and interpreted data, and drafted the manuscript. MS, TS and YI acquired clinical data. KJ, OW and TW analyzed and interpreted imaging data. HA and OS supervised and critically edited the manuscript. All authors read and approved the final manuscript.

\section{Acknowledgements}

This work was supported by funding from a Grant-in-Aid for Scientific Research (C) 23592329 and 26462407 from The Ministry of Education, Culture, Sports, Science and Technology of Japan.

\section{Author details}

'Departments of Urology, Hamamatsu University School of Medicine, 1-20-1 Higashi-ku, Handayama, Hamamatsu, Shizuoka 431-3192, Japan.

${ }^{2}$ Departments of Radiology, Hamamatsu University School of Medicine, 1-20-1 Higashi-ku, Handayama, Hamamatsu, Shizuoka 431-3192, Japan. ${ }^{3}$ Department of Medical Physics and Radiology, University of Wisconsin-Madison, 600 Highland Ave, CSC, Madison, WI 53792, USA. ${ }^{4}$ Applied Science Laboratory Asia Pacific, GE Healthcare Japan, 4-7-127, Asahigaoka, Hino, Tokyo 191-8503, Japan.

Received: 22 January 2014 Accepted: 11 August 2014

Published: 14 August 2014

\section{References}

1. Motzer RJ, Hutson TE, Tomczak P, Michaelson MD, Bukowski RM, Oudard S, Negrier S, Szczylik C, Pili R, Bjarnason GA, Garcia-del-Muro X, Sosman JA, Solska E, Wilding G, Thompson JA, Kim ST, Chen I, Huang X, Figlin RA: Overall survival and updated results for sunitinib compared with interferon alfa in patients with metastatic renal cell carcinoma. J Clin Oncol 2009, 27:3584-3590.

2. Escudier B, Bellmunt J, Négrier S, Bajetta E, Melichar B, Bracarda S, Ravaud A, Golding S, Jethwa S, Sneller V: Phase III trial of bevacizumab plus interferon alfa-2a in patients with metastatic renal cell carcinoma (AVOREN): final analysis of overall survival. J Clin Oncol 2010, 28:2144-2150.

3. Sternberg CN, Davis ID, Mardiak J, Szczylik C, Lee E, Wagstaff J, Barrios CH, Salman P, Gladkov OA, Kavina A, Zarbá JJ, Chen M, McCann L, Pandite L, Roychowdhury DF, Hawkins RE: Pazopanib in locally advanced or metastatic renal cell carcinoma: results of a randomized phase III trial. J Clin Oncol 2010, 28:1061-1068.

4. Hudes G, Carducci M, Tomczak P, Dutcher J, Figlin R, Kapoor A, Staroslawska E, Sosman J, McDermott D, Bodrogi I, Kovacevic Z, Lesovoy V, Schmidt-Wolf IG, Barbarash O, Gokmen E, OToole T, Lustgarten S, Moore L, Motzer RJ: Global ARCC Trial: Temsirolimus, interferon-alpha or both for advanced renal cell carcinoma. N Engl J Med 2007, 356:2271-2281.

5. Choi H, Charnsangavej C, de Castro Faria S, Tamm EP, Benjamin RS, Johnson MM, Macapinlac HA, Podoloff DA: CT evaluation of the response of gastrointestinal stromal tumors after imatinib mesylate treatment: a quantitative analysis correlated with FDG PET findings. AJR Am J Roentgenol 2004, 183:1619-1628. 
6. Ueno D, Yao M, Tateishi U, Minamimoto R, Makiyama K, Hayashi N, Sano F, Murakami T, Kishida T, Miura T, Kobayashi K, Noguchi S, Ikeda I, Ohgo Y, Inoue T, Kubota Y, Nakaigawa N: Early assessment by FDG-PET/CT of patients with advanced renal cell carcinoma treated with tyrosine kinase inhibitors is predictive of disease course. BMC Cancer 2012, 12:162.

7. Khandani AH, Cowey CL, Moore DT, Gohil H, Rathmell WK: Primary renal cell carcinoma: relationship between 18 F-FDG uptake and response to neoadjuvant sorafenib. Nucl Med Commun 2012, 33:967-973.

8. François CJ, Lum DP, Johnson KM, Landgraf BR, Bley TA, Reeder SB, Schiebler ML, Grist TM, Wieben O: Renal arteries: isotropic, high-spatialresolution, unenhanced MR angiography with three-dimensional radial phase contrast. Radiology 2011, 258:254-260.

9. Bernstein EJ, Schmidt-Lauber C, Kay J: Nephrogenic systemic fibrosis: a systemic fibrosing disease resulting from gadolinium exposure. Best Pract Res Clin Rheumatol 2012, 26:489-503.

doi:10.1186/1756-0500-7-527

Cite this article as: Takayama et al:: Use of three-dimensional time-resolved phase-contrast magnetic resonance imaging with vastly undersampled isotropic projection reconstruction to assess renal blood flow in a renal cell carcinoma patient treated with sunitinib: a case report. BMC Research Notes 2014 7:527.

\section{Submit your next manuscript to BioMed Central and take full advantage of:}

- Convenient online submission

- Thorough peer review

- No space constraints or color figure charges

- Immediate publication on acceptance

- Inclusion in PubMed, CAS, Scopus and Google Scholar

- Research which is freely available for redistribution 